\title{
High IKBIP expression as a biomarker for poor prognosis and correlated with immune infiltrates in hepatocellular carcinoma (HCC)
}

\section{Mingxin Lin ( $\square$ mingxinlin@126.com )}

Xiamen University School of Medicine

Jingping Liu

Jiangsu Province Hospital and Nanjing Medical University First Affiliated Hospital

\section{Xiaoli Shi}

Jiangsu Province People's Hospital and Nanjing Medical University First Affiliated Hospital: Jiangsu

Province Hospital and Nanjing Medical University First Affiliated Hospital

\section{Jiajun Li}

Huazhong University of Science and Technology Tongji Medical College First Clinical College: Wuhan Union Hospital

Huiming Ye

Xiamen University School of Public Health

\section{Research Article}

Keywords: IKBIP, hepatocellular carcinoma, biomarker, prognosis, immune infiltrates

Posted Date: November 18th, 2021

DOI: https://doi.org/10.21203/rs.3.rs-1038089/v1

License: (9) This work is licensed under a Creative Commons Attribution 4.0 International License. Read Full License 


\section{Abstract}

Background: I kappa B kinase interacting protein (IKBIP) is dysregulated and closely correlated with prognosis in tumor. However, the potential functions of IKBIP have not been utterly revealed in hepatocellular carcinoma (HCC). Thus, we explored the clinical values of IKBIP and its correlation with immune infiltrates in HCC.

Methods: IKBIP expression in different tumor types was analyzed through the Tumor Immunity Evaluation Resource (TIMER) database. IKBIP expression between HCC and normal tissues were readily available for retrospective analyses from GEPIA, UALCAN and the Cancer Genome Atlas (TCGA) databases. The association between clinical features and IKBIP expression in HCC was analyzed by the Wilcoxon signed-rank test and logistic regression. Human Protein Atlas (HPA) was used to analyze the protein expression of IKBIP. A receiver operating characteristic (ROC) curve was performed to explore diagnostic potential of IKBIP. Kaplan-Meier (K-M) method and Cox regression were conducted to evaluate the influence of IKBIP on the prognosis of HCC patients. Protein-protein interaction (PPI) networks were established using STRING. Gene ontology (GO) and Kyoto Encyclopedia of Genes and Genomes (KEGG) were used for gene functional enrichment analysis. The interrelation between IKBIP expression and immune infiltrates in HCC was analyzed through TIMER and tumor-immune system interaction database (TISIDB).

Results: Our results revealed that IKBIP expression was abnormally upregulated in $\mathrm{HCC}$, and ROC curve analysis confirmed the diagnostic power of IKBIP. High IKBIP expression was related to tumor sizes, pathologic stage and histologic grade. K-M plotter analysis showed that high IKBIP expression was correlated with poor prognosis of HCC patients. Moreover, multivariate Cox analysis further verified that high IKBIP expression was an independent risk factor in HCC patients. Correlation analysis found that IKBIP expression was associated with infiltrating immune cells.

Conclusion: High IKBIP expression is associated with poor prognosis and immune infiltrates, which may serve as a prognostic biomarker and therapeutic target for HCC.

\section{Introduction}

Hepatocellular carcinoma (HCC), approximately accounting for $75 \%-85 \%$ of all primary liver malignancies, is one of the leading causes of cancer-related mortality around the world (1-3). In recent years, new targeted and immune therapies could improve overall survival and improve quality of life in HCC patients. However, the prognosis still remains unsatisfactory, and the 5-year survival rate is only $7 \%$ (4-6). Thus, more potential molecular targets are urgently needed for improving the treatment of HCC patients.

I kappa B kinase interacting protein (IKBIP) was found to be located on human chromosome 12. HoferWarbinek et al. showed that IKBIP was a target gene of $p 53$ and promoted the apoptosis (7). A recent paper reported that IKBIP was upregulated and closely correlated with aggressive phenotypes in glioma. 
High IKBIP expression was correlated with poor prognosis of patients with glioma. Moreover, IKBIP was closely related to a series of EMT-related biological processes, which indicated that IKBIP might contribute to tumorigenesis and progression of glioma through EMT induction(8). However, the potential functions of IKBIP in HCC still remain unclear. We hypothesized that, similar to glioma, the level of IKBIP is closely related to survival in HCC. Thus, we further explored the diagnostic and prognostic values of IKBIP in HCC and its correlation with immune infiltration based on multiple databases in this study.

\section{Materials And Methods}

\section{Analysis of Tumor Immune Estimation Resource (TIMER) database}

TIMER (https://cistrome.shinyapps.io/timer/) is a comprehensive portal for the systematic assessment of immune infiltrations in different tumor types (9). In this study, IKBIP expression in diverse cancer types was analyzed via the "Diff Exp" module. Additionally, the "Gene" module was used to investigate the relationship between IKBIP expression and tumor-infiltrating immune cells (TILs).

\section{Analysis of the Cancer Genome Atlas (TCGA) database}

The IKBIP expression data and relevant clinical information in HCC patients were obtained from TCGA website (http://cancergenome.nih.gov/)(10). Besides, we eliminated any samples with duplicate or insufficient information in this study.

\section{Analysis of GEPIA and UALCAN databases}

GEPIA (http://gepia.cancerpku.cn/) and UALCAN (http://ualcan.path.uab.edu) were the online databases to analyzed gene expression data from TCGA $(11,12)$. In this study, we utilized GEPIA and UALCAN databases to analyze IKBIP expression in HCC and normal tissues.

\section{Analysis of Human Protein Atlas (HPA)}

The protein expression of normal and tumor tissues was assessed via HPA(13). Thus, we used HPA to analyze the protein expression of IKBIP in HCC and tumor tissues.

\section{Analysis of Kaplan-Meier (K-M) plotter database}

The K-M plotter (http://kmplot.com/analysis/) can assess the effect of various biomolecules in tumor on the survival rate(14). In this study, the $\mathrm{K}-\mathrm{M}$ plotter was performed to assess the prognostic potential of IKBIP in HCC patients.

\section{Analysis of Protein-Protein Interaction (PPI) and gene functional enrichment analysis}

The PPI network of IKBIP was established by STRING online database (https://string-db.org/)(15).

Meanwhile, Gene ontology (GO) enrichment and Kyoto Encyclopedia of Genes and Genomes (KEGG) were 
used for analyzing the possible biological functions of IKBIP.

\section{Analysis of Tumor-Immune System Interaction database (TISIDB)}

TISIDB is an online web for analyzing tumor-immune system interaction(16). We utilized TISIDB to analyze IKBIP expression and TILs in various cancers. Meanwhile, the interrelations between IKBIP expression and TILs were also evaluated by TISIDB.

\section{Statistical Analyses}

Mann-Whitney U-test and Paired $t$-test were performed to compare IKBIP expression levels between HCC and normal tissues. The ROC curve was conducted to assess the diagnostic potential of IKBIP. Wilcoxon signed-rank test and logistic regression were performed to analyze the correlation between clinical features and IKBIP expression in HCC. K-M plotter was used for assessing the influence of IKBIP on survival. Univariate and multivariate Cox analyses were performed to explore the potential prognostic factors in HCC. The nomogram and calibration curves were conducted to predict 1-, 3- and 5-year overall survival (OS) for HCC patients. $P$ value $<0.05$ was considered as statistically significant.

\section{Results}

\section{IKBIP expression was elevated in HCC and other cancers}

IKBIP expression in various types of cancer was first analyzed using TIMER database. Higher expression of IKBIP was significantly elevated in bladder urothelial carcinoma (BLCA), breast invasive carcinoma (BRCA), cholangiocarcinoma ( $\mathrm{CHOL})$, colon adenocarcinoma (COAD), esophageal carcinoma (ESCA), head and neck squamous cell carcinoma (HNSC), kidney chromophobe $(\mathrm{KICH})$, kidney renal clear cell carcinoma (KIRC), kidney renal papillary cell carcinoma (KIRP), liver hepatocellular carcinoma (LIHC), lung adenocarcinoma (LUAD), lung squamous cell carcinoma (LUSC), pheochromocytoma and paraganglioma (PCPG), prostate adenocarcinoma (PRAD), rectum adenocarcinoma (READ), stomach adenocarcinoma (STAD), thyroid carcinoma (THCA) and uterine corpus endometrial carcinoma (UCEC) compared with the corresponding normal tissues (Figure 1). Further studies showed that IKBIP expression in HCC patients was also elevated in GEPIA and UALCAN databases (Figure 2A-B). Moreover, we found consistent results in the TCGA database (Figure 2C), and paired data analyses indicated that IKBIP expression in HCC tissues ( $n=50)$ was significantly higher than those in normal tissues $(n=50)$ (Figure 2D). Besides, we found that IKBIP protein expression was upregulated in HCC tissue by immunohistochemical staining from HPA (Figure 2E). In TCGA database, the ROC analysis suggested that the level of IKBIP expression had a diagnostic potential in HCC patients (Figure 2F).

\section{Correlations between IKBIP expression and clinical features in HCC patients}

The clinical features and gene expression data of HCC patients were obtained from TCGA database. Wilcoxon signed-rank test and logistic regression were performed to evaluate the association between 
IKBIP expression and clinical features. High IKBIP expression was significantly correlated with $\mathrm{T}$ stage $(P$ $<0.001)$, pathologic stage $(P<0.001)$ and histologic grade $(P=0.002)$ (Table 1 and Figures $3 \mathrm{~A}-\mathrm{H})$. While it was not associated with other features, including age $(P=0.055)$, gender $(P=0.377), \mathrm{N}$ stage $(P=$ $0.623)$, M stage $(P=1.000)$ and residual tumor $(P=0.898)$. The univariate analysis indicated that IKBIP expression was correlated with the poor prognostic for HCC patients. High IKBIP expression was significantly associated with $\mathrm{T}$ stage (T3\&T4 vs T1\&T2: $\mathrm{OR}=2.484,95 \% \mathrm{Cl}=1.530-4.100, P<0.001$ ), pathologic stage (Stage III\&Stage IV vs. Stage I\&Stage II: OR $=2.643,95 \% \mathrm{Cl}=1.611-4.411, P<0.001$ ) and histologic grade (G3\&G4 vs. G1\&G2: OR = 2.148, 95\% Cl=1.399-3.324, $P<0.001)$ (Table 2). 
Table 1

Correlation between IKBIP expression and clinical features of patients with HCC (TCGA).

\begin{tabular}{|c|c|c|c|}
\hline \multirow[t]{2}{*}{ Characteristic } & Low IKBIP expression & High IKBIP expression & \multirow[t]{2}{*}{$P$} \\
\hline & $\mathrm{N}(\%)$ & $\mathrm{N}(\%)$ & \\
\hline \multicolumn{3}{|l|}{ Age } & \multirow[t]{3}{*}{0.055} \\
\hline$<=60$ & $79(21.2 \%)$ & $98(26.3 \%)$ & \\
\hline$>60$ & $108(29 \%)$ & $88(23.6 \%)$ & \\
\hline \multicolumn{3}{|l|}{ Gender } & \multirow[t]{3}{*}{0.377} \\
\hline Female & $56(15 \%)$ & $65(17.4 \%)$ & \\
\hline Male & $131(35 \%)$ & $122(32.6 \%)$ & \\
\hline \multicolumn{3}{|l|}{ T stage } & \multirow[t]{5}{*}{$<0.001$} \\
\hline T1 & $110(29.6 \%)$ & $73(19.7 \%)$ & \\
\hline T2 & $44(11.9 \%)$ & $51(13.7 \%)$ & \\
\hline T3 & $29(7.8 \%)$ & $51(13.7 \%)$ & \\
\hline T4 & $2(0.5 \%)$ & $11(3 \%)$ & \\
\hline \multicolumn{3}{|l|}{ N stage } & \multirow[t]{3}{*}{0.623} \\
\hline No & $123(47.7 \%)$ & $131(50.8 \%)$ & \\
\hline N1 & $1(0.4 \%)$ & $3(1.2 \%)$ & \\
\hline \multicolumn{3}{|l|}{ M stage } & \multirow[t]{3}{*}{1.000} \\
\hline MO & $133(48.9 \%)$ & 135 (49.6\%) & \\
\hline M1 & $2(0.7 \%)$ & $2(0.7 \%)$ & \\
\hline \multicolumn{3}{|l|}{ Pathologic stage } & \multirow[t]{5}{*}{$<0.001$} \\
\hline Stage I & $105(30 \%)$ & $68(19.4 \%)$ & \\
\hline Stage II & $43(12.3 \%)$ & $44(12.6 \%)$ & \\
\hline Stage III & $27(7.7 \%)$ & $58(16.6 \%)$ & \\
\hline Stage IV & $3(0.9 \%)$ & $2(0.6 \%)$ & \\
\hline \multicolumn{3}{|l|}{ Residual tumor } & \multirow[t]{3}{*}{0.898} \\
\hline Ro & $171(49.6 \%)$ & $156(45.2 \%)$ & \\
\hline R1 & $8(2.3 \%)$ & $9(2.6 \%)$ & \\
\hline
\end{tabular}




\begin{tabular}{|c|c|c|c|}
\hline R2 & $1(0.3 \%)$ & $0(0 \%)$ & \\
\hline \multicolumn{3}{|c|}{ Histologic grade } & \multirow[t]{5}{*}{0.002} \\
\hline G1 & $36(9.8 \%)$ & $19(5.1 \%)$ & \\
\hline G2 & $97(26.3 \%)$ & $81(22 \%)$ & \\
\hline G3 & 46 (12.5\%) & $78(21.1 \%)$ & \\
\hline G4 & $6(1.6 \%)$ & $6(1.6 \%)$ & \\
\hline
\end{tabular}

Table 2

IKBIP expression associated with clinicopathologic features using logistic regression

\begin{tabular}{|llll|}
\hline Characteristics & Total & Odds Ratio (OR) & $\begin{array}{l}P \\
\text { value }\end{array}$ \\
\hline Age (>60 vs. <=60) & 373 & $0.657(0.436-0.987)$ & 0.044 \\
\hline Gender (Male vs. Female) & 374 & $0.802(0.519-1.238)$ & 0.320 \\
\hline T stage (T3\&T4 vs. T1\&T2) & 371 & $2.484(1.530-4.100)$ & $<0.001$ \\
\hline N stage (N1 vs. N0) & 258 & $2.817(0.355-$ & 0.373 \\
\hline M stage (M1 vs. M0) & 272 & $0.985(0.117-8.308)$ & 0.988 \\
\hline $\begin{array}{l}\text { Pathologic stage (Stage III\&Stage IV vs. Stage I\&Stage } \\
\text { II) }\end{array}$ & 350 & $2.643(1.611-4.411)$ & $<0.001$ \\
\hline Residual tumor (R1\&R2 vs. R0) & 345 & $1.096(0.418-2.876)$ & 0.850 \\
\hline Histologic grade (G3\&G4 vs. G1\&G2) & 369 & $2.148(1.399-3.324)$ & $<0.001$ \\
\hline
\end{tabular}

\section{The independent risk factor and prognostic potential of IKBIP expression in HCC patients}

The survival analysis demonstrated that high expression of IKBIP was related to poor OS $(P=0.002)$ (Figure 4A), and the K-M plotter showed that high IKBIP expression was also associated with poor OS ( $P=$ 3.4e-05) (Figure 4B), poor progression-free survival (PFS) $(P=8.7 \mathrm{e}-0.5)$ (Figure 4C) and poor disease-free survival (DFS) $(P<0.001)$ (Figure 4D). In multivariate Cox analysis, $\mathrm{T}$ stage $(\mathrm{HR}=0.394,95 \% \mathrm{Cl}=10.277$ $0.560, P<0.001)$, pathologic stage $(\mathrm{HR}=0.408,95 \% \mathrm{Cl}=0.282-0.592, P<0.001)$, and IKBIP expression $(\mathrm{HR}=1.543,95 \% \mathrm{Cl}=1.256-1.896, P<0.001)$ were significantly correlated with poor OS. Moreover, multivariate regression analysis further confirmed that only IKBIP expression was an independent prognostic factor in $\mathrm{HCC}(\mathrm{HR}=1.506,95 \% \mathrm{Cl}=11.149-1.973, P<0.01)$ (Table 3). Besides, the nomogram was conducted to predict the 1, 3, 5-year OS of HCC patients (Figure 5A), and the accuracy of prediction was evaluated by Calibration curve (Figure 5B). 
Table 3

Associations with clinical features in HCC patients using cox regression.

\begin{tabular}{|c|c|c|c|c|c|}
\hline \multirow[t]{2}{*}{ Characteristics } & \multirow[t]{2}{*}{ Total(N) } & \multicolumn{2}{|c|}{ Univariate analysis } & \multicolumn{2}{|c|}{$\begin{array}{l}\text { Multivariate } \\
\text { analysis }\end{array}$} \\
\hline & & $\begin{array}{l}\text { Hazard ratio } \\
(95 \% \mathrm{Cl})\end{array}$ & $\begin{array}{l}P \\
\text { value }\end{array}$ & $\begin{array}{l}\text { Hazard } \\
\text { ratio } \\
(95 \% \mathrm{Cl})\end{array}$ & $\begin{array}{l}\mathrm{P} \\
\text { value }\end{array}$ \\
\hline Gender (Female vs Male) & 370 & $\begin{array}{l}0.816 \\
(0.573- \\
1.163)\end{array}$ & 0.260 & & \\
\hline Age (>60 vs<=60) & 370 & $\begin{array}{l}1.248 \\
(0.880- \\
1.768)\end{array}$ & 0.214 & & \\
\hline T stage (T3\&T4 vs T1\&T2) & 367 & $\begin{array}{l}0.394 \\
(0.277- \\
0.560)\end{array}$ & $<0.001$ & $\begin{array}{l}0.350 \\
(0.046- \\
2.676)\end{array}$ & 0.312 \\
\hline N stage (N1 vs N0) & 256 & $\begin{array}{l}0.499 \\
(0.122- \\
2.037)\end{array}$ & 0.333 & & \\
\hline M stage (M1 vs M0) & 270 & $\begin{array}{l}0.248 \\
(0.078- \\
0.789)\end{array}$ & 0.018 & $\begin{array}{l}0.401 \\
(0.121- \\
1.321)\end{array}$ & 0.133 \\
\hline $\begin{array}{l}\text { Pathologic stage (Stage III \& Stage IV } \\
\text { vs Stage I \& Stage II) }\end{array}$ & 346 & $\begin{array}{l}0.408 \\
(0.282- \\
0.592)\end{array}$ & $<0.001$ & $\begin{array}{l}1.175 \\
(0.152- \\
9.102)\end{array}$ & 0.877 \\
\hline Residual tumor (R1\&R2 vs R0) & 341 & $\begin{array}{l}0.637 \\
(0.322- \\
1.258)\end{array}$ & 0.194 & & \\
\hline Histologic grade (G3\&G4 vs G1\&G2) & 365 & $\begin{array}{l}0.893 \\
(0.623- \\
1.281)\end{array}$ & 0.539 & & \\
\hline IKBIP & 370 & $\begin{array}{l}1.543 \\
(1.256- \\
1.896)\end{array}$ & $<0.001$ & $\begin{array}{l}1.506 \\
(1.149- \\
1.973)\end{array}$ & 0.003 \\
\hline
\end{tabular}

\section{PPI networks and gene functional enrichment analysis}

We explored PPI networks and gene functional enrichment by using STRING database, GO and KEGG analyses. As shown in Figure 6A-B, the network of IKBIP, GO and KEGG analyses indicated IKBIP was correlated with endocytosis, Golgi-associated vesicle membrane, coated membrane, membrane coat and vesicle coat. The interrelation analyses between IKBIP expression and 10 co-expressed genes were shown in Figure 6C-L. 
We utilized TIMER database to analyze the association between IKBIP expression and TILs. IKBIP expression was associated with tumor purity $(r=-0067, P=2.11 \mathrm{e}-01)$, B cell $(r=0.347, P=3.45 \mathrm{e}-11)$, $\mathrm{CD}^{+} \mathrm{T}$ cell $(\mathrm{r}=0.368, P=2.15 \mathrm{e}-12), \mathrm{CD}^{+} \mathrm{T}$ cell $(\mathrm{r}=0.328, P=4.79 \mathrm{e}-10)$, macrophage $(\mathrm{r}=0.486, P=$ 1.34e-21), neutrophil ( $r=0.425, P=1.33 \mathrm{e}-16)$, dendritic cell $(r=0.446, P=5.42 \mathrm{e}-18)$ (Figure 7A).

Moreover, we also used TISIDB database to evaluate the interrelation between IKBIP expression and TILs (Figure 7B), and the findings indicated IKBIP expression was related to the Act-CD4 ${ }^{+} \mathrm{T}$ cells $(\mathrm{r}=0.479, P<$ 2.2e-16), Tem-CD4 ${ }^{+} \mathrm{T}$ cells $(r=0.256, P=5.88 \mathrm{e}-07)$, NKT cells $(r=0.222, P=1.6 \mathrm{e}-05)$ and Th2 cells $(r=$ $0.346, P=8.46 \mathrm{e}-12$ ) (Figure 7C).

\section{Discussion}

$\mathrm{HCC}$ has become a major health problem worldwide with the characteristics of invasion, metastasis and frequent recurrence $(17,18)$. The treatment of HCC has made great progress, but the prognosis of HCC still remains unsatisfactory(19). Thus, it is necessary to explore new immunotherapy targets for HCC. IKBIP is located on chromosome 12, which was described as the target of $p 53$ with pro-apoptotic activity(20). A recent paper reported that IKBIP was upregulated in glioma and correlated with an unsatisfactory prognosis (21). However, the clinical values of IKBIP have not been utterly revealed in HCC.

In this study, our results found that IKBIP was elevated in various cancers by pan-cancer analysis. Moreover, we also confirmed that IKBIP expression in HCC was significantly upregulated in multiple databases. The IKBIP expression was associated with tumor sizes, high pathologic stage and histologic grade. ROC curve analysis found that IKBIP serves as a promising diagnostic biomarker in HCC patients. Based on K-M plotter and univariate analysis, we found that high IKBIP expression was related to short OS, PFS and DFS. Univariate Cox analysis further confirmed that the level of IKBIP was risk factors for the prognosis in HCC. These results indicated that IKBIP might serve as a novel prognostic factor and a potential new target for the treatment of HCC patients.

Recent studies have demonstrated that IKBIP could promote the occurrence and development of tumor by Epithelial-to-mesenchymal transition (EMT) induction(8). EMT plays a crucial role not only in promoting tumor invasion and migration, but also in tumor recurrence and treatment resistance(22, 23). Besides, IKBIP has also been shown to be related to immune and inflammatory responses (24). Based on these, we conclude that IKBIP may play an immunosuppressive role in tumor. However, the biological function of IKBIP has not been investigated in HCC. Thus, we further elucidated the function of IKBIP in HCC. We found that Hub genes of IKBIP in PPI network were apoptotic protease-activating factor 1 (APAF1), GTPase-activating protein 3 (ARFGAP3), clathrin light chain B (CLTB), NFU1, protein kinase C and casein kinase substrate in neurons 2 (PACSIN2), patched domain containing 3 (PTCHD3), RAB11 family interacting protein 1 (RAB11FIP1), RPUSD4, serine/arginine-rich splicing factor 7 (SRSF7) and TTLL1. GO and KEGG analysis indicated that KIBIP and its co-expressed genes were enriched in endocytosis, Golgiassociated vesicle membrane, coated membrane, membrane coat and vesicle coat. Among co-expressed genes, ARFGAP3, a GTPase-activating protein, which was correlated with the Golgi-apparatus and involve in the vesicular trafficking pathway, and it has been confirmed that ARFGAP3 could promote the 
proliferation and migration of prostate cancer cell(25). RAB11FIP1, also known as Rab-coupling protein (RCP), which was involved in tumor invasion and metastasis in breast cancer(26). SRSF7 was frequently upregulated in colon and lung cancer, which supported that SRSF7 might serve as a proto-oncogene(27). These findings indicated IKBIP might be involved in the occurrence and development of tumors by synergizing other tumor-related genes.

In recent years, the role of the immune system in cancer has been received increasing attention with the wide application of tumor immunotherapy $(28,29)$. Accumulating studies demonstrated that immune cells in the tumor microenvironment (TME) contribute to tumor occurrence and progression (30). Therefore, we explored the interrelation between IKBIP expression and TILs. In this study, we found that IKBIP expression was closely correlated with to six tumor infiltrating immune cells (B cell, $\mathrm{CD}^{+} \mathrm{T}^{-}$cell, $\mathrm{CD}^{+} \mathrm{T}$ cell, macrophage, neutrophil, dendritic cell) in HCC. Besides, we also found IKBIP expression was positively correlated with Act-CD4 ${ }^{+} \mathrm{T}$ cells, Tem-CD4 ${ }^{+} \mathrm{T}$ cells, NKT cells and Th2 cells. Our results suggested that there was a potential interrelation between IKBIP and immune infiltration cells in HCC. Nevertheless, relevant research should be explored to verify the above findings in future studies.

There are some limitations to address in this study. First, IKBIP expression and its prognostic value were analyzed by online public databases, which should be required to be validated in large-scale clinical samples. Second, the vivo/vitro experiments are needed to further explore the influence of IKBIP on the immune infiltrations in HCC.

In conclusion, we first found that IKBIP expression was upregulated and correlated with tumor sizes, pathologic stage and histologic grade in HCC. Moreover, IKBIP expression was also closely associated with the poor prognosis and immune infiltrations. Therefore, our findings suggested that IKBIP could serve as a potential biomarker for poor prognosis and might play a crucial role in immune infiltrations in HCC.

\section{Abbreviations}

HCC: hepatocellular carcinoma; IKBIP: I kappa B kinase interacting protein; TIMER: Tumor Immunity Evaluation Resource; TCGA: The Cancer Genome Atlas; K-M: Kaplan-Meier; HPA: Human Protein Atlas; ROC: Receiver operating characteristic; PPI: Protein-protein interaction; TISIDB: Tumor-immune system interaction database; TILs: Tumor-infiltrating immune cells; OS: Overall survival; PFS: Progression-free survival; DFS: Disease-free survival; BLCA: Bladder urothelial carcinoma; BRCA: Breast invasive carcinoma; CHOL: Cholangiocarcinoma; COAD: Colon adenocarcinoma; ESCA: Esophageal carcinoma; HNSC: Head and neck squamous cell carcinoma; KICH: Kidney chromophobe; KIRC Kidney renal clear cell carcinoma; KIRP: Kidney renal papillary cell carcinoma; LIHC: Liver hepatocellular carcinoma; LUAD: Lung adenocarcinoma; LUSC: Lung squamous cell carcinoma; PCPG: Pheochromocytoma and Paraganglioma; PRAD: Prostate adenocarcinoma; READ: Rectum adenocarcinoma; STAD: Stomach adenocarcinoma; THCA: Thyroid carcinoma; UCEC: uterine corpus endometrial carcinoma; EMT: Epithelial-to-mesenchymal transition; APAF1: Apoptotic protease-activating factor 1; ARFGAP3: GTPase-activating protein 3; CLTB: 
Clathrin light chain B; PACSIN2: Protein kinase $C$ and casein kinase substrate in neurons 2; PTCHD3:

Patched domain containing 3; RAB11FIP1: RAB11 family interacting protein 1; SRSF7: Serine/argininerich splicing factor 7 .

\section{Declarations}

\section{Acknowledgements}

The authors thank all individuals who participated in this study

\section{Authors' contributions}

$\mathrm{ML}$ and $\mathrm{HY}$ contributed to the design of the study. $\mathrm{ML}$, JL and XS contributed to the statistical analysis. $\mathrm{JL}$ contributed to making diagrams. All authors read and approved the final manuscript.

\section{Funding}

Not applicable.

\section{Availability of data and materials}

The data that support the findings of this study are available from the corresponding author upon reasonable request.

\section{Ethics approval and consent to participate}

The study complied with the principles set forth in the Declaration of Helsinki. Access to the relevant data was obtained from the TIMER, GEPIA, UALCAN, TCGA, STING and TISIDB databases following the database policy. Ethics approval were not required.

\section{Consent for publication}

Not applicable.

\section{Competing interests}

The authors declare that they have no competing interests.

\section{References}

1. Petrick JL, Florio AA, Znaor A, et al. International trends in hepatocellular carcinoma incidence, 19782012. Int J Cancer. 2020;147(2):317-30.

2. Yang JD, Heimbach JK. New advances in the diagnosis and management of hepatocellular carcinoma. Bmj. 2020;371:m3544. 
3. Sung H, Ferlay J, Siegel RL, et al. Global Cancer Statistics 2020: GLOBOCAN Estimates of Incidence and Mortality Worldwide for 36 Cancers in 185 Countries. CA Cancer J Clin. 2021;71(3):209-49.

4. Neureiter D, Stintzing S, Kiesslich T, et al. Hepatocellular carcinoma: Therapeutic advances in signaling, epigenetic and immune targets. World journal of gastroenterology. 2019;25(25):3136-50.

5. Vanderborght B, Lefere S, Vlierberghe HV, et al. The Angiopoietin/Tie2 Pathway in Hepatocellular Carcinoma. Cells. 2020;9(11).

6. Zheng Y, Zhu M, Li M. Effects of alpha-fetoprotein on the occurrence and progression of hepatocellular carcinoma. Journal of cancer research and clinical oncology. 2020;146(10):2439-46.

7. Hofer-Warbinek R, Schmid JA, Mayer $\mathrm{H}$, et al. A highly conserved proapoptotic gene, IKIP, located next to the APAF1 gene locus, is regulated by p53. Cell death and differentiation. 2004;11(12):1317-25.

8. Yang Y, Wang J, Xu S, et al. IKBIP is a novel EMT-related biomarker and predicts poor survival in glioma. Translational neuroscience. 2021;12(1):9-19.

9. Li T, Fan J, Wang B, et al. TIMER: A Web Server for Comprehensive Analysis of Tumor-Infiltrating Immune Cells. Cancer research. 2017;77(21):e108-e10.

10. Wang Z, Jensen MA, Zenklusen JC. A Practical Guide to The Cancer Genome Atlas (TCGA). Methods in molecular biology. 2016;1418:111-41.

11. Tang Z, Li C, Kang B, et al. GEPIA: a web server for cancer and normal gene expression profiling and interactive analyses. Nucleic acids research. 2017;45(W1):W98-W102.

12. Chandrashekar DS, Bashel B, Balasubramanya SAH, et al. UALCAN: A Portal for Facilitating Tumor Subgroup Gene Expression and Survival Analyses. Neoplasia. 2017;19(8):649-58.

13. Digre A, Lindskog C. The Human Protein Atlas-Spatial localization of the human proteome in health and disease. Protein science: a publication of the Protein Society. 2021;30(1):218-33.

14. Xie XW, Jiang SS, Li X. CLEC3B as a Potential Prognostic Biomarker in Hepatocellular Carcinoma. Frontiers in molecular biosciences. 2020;7:614034.

15. Szklarczyk D, Morris JH, Cook H, et al. The STRING database in 2017: quality-controlled proteinprotein association networks, made broadly accessible. Nucleic acids research. 2017;45(D1):D362D8.

16. Ru B, Wong CN, Tong Y, et al. TISIDB: an integrated repository portal for tumor-immune system interactions. Bioinformatics. 2019;35(20):4200-2.

17. Chen L, Guo P, He Y, et al. HCC-derived exosomes elicit HCC progression and recurrence by epithelialmesenchymal transition through MAPK/ERK signalling pathway. Cell death \& disease. 2018;9(5):513.

18. Jiang H, Zhou Z, Jin S, et al. PRMT9 promotes hepatocellular carcinoma invasion and metastasis via activating PI3K/Akt/GSK-3beta/Snail signaling. Cancer science. 2018;109(5):1414-27.

19. Zhang H, Ye Y, Li W. Perspectives of Molecular Therapy-Targeted Mitochondrial Fission in Hepatocellular Carcinoma. Biomed Res Int. 2020;2020:1039312. 
20. Long S, Li G. Comprehensive analysis of a long non-coding RNA-mediated competitive endogenous RNA network in glioblastoma multiforme. Experimental and therapeutic medicine. 2019;18(2):108190.

21. Chen TY, Liu Y, Chen L, et al. Identification of the potential biomarkers in patients with glioma: a weighted gene co-expression network analysis. Carcinogenesis. 2020;41(6):743-50.

22. Pastushenko I, Blanpain C. EMT Transition States during Tumor Progression and Metastasis. Trends in cell biology. 2019;29(3):212-26.

23. Aiello NM, Kang Y. Context-dependent EMT programs in cancer metastasis. J Exp Med. 2019;216(5):1016-26.

24. Wu H, Liu H, Zhao X, et al. IKIP Negatively Regulates NF-kappaB Activation and Inflammation through Inhibition of IKKalpha/beta Phosphorylation. J Immunol. 2020;204(2):418-27.

25. Obinata $D$, Takayama $K$, Urano $T$, et al. ARFGAP3, an androgen target gene, promotes prostate cancer cell proliferation and migration. Int J Cancer. 2012;130(10):2240-8.

26. Takao C, Morikawa A, Ohkubo H, et al. Downregulation of ARID1A, a component of the SWI/SNF chromatin remodeling complex, in breast cancer. Journal of Cancer. 2017;8(1):1-8.

27. Fu Y, Wang Y. SRSF7 knockdown promotes apoptosis of colon and lung cancer cells. Oncol Lett. 2018;15(4):5545-52.

28. Lei X, Lei Y, Li JK, et al. Immune cells within the tumor microenvironment: Biological functions and roles in cancer immunotherapy. Cancer letters. 2020;470:126-33.

29. Petitprez F, Meylan M, de Reynies A, et al. The Tumor Microenvironment in the Response to Immune Checkpoint Blockade Therapies. Front Immunol. 2020;11:784.

30. Hinshaw DC, Shevde LA. The Tumor Microenvironment Innately Modulates Cancer Progression. Cancer research. 2019;79(18):4557-66.

\section{Figures}




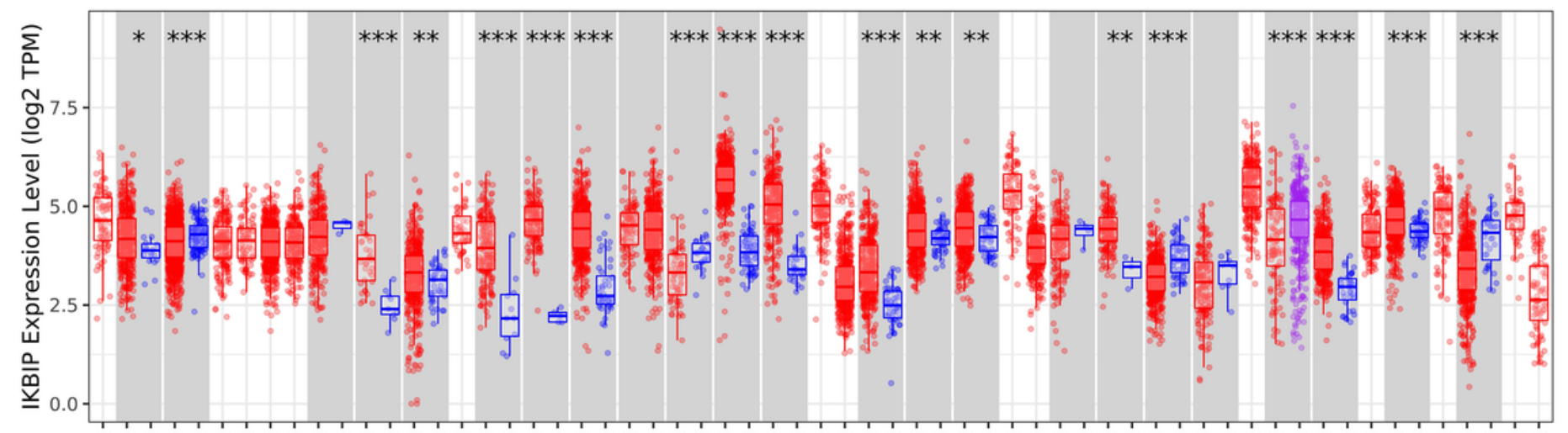

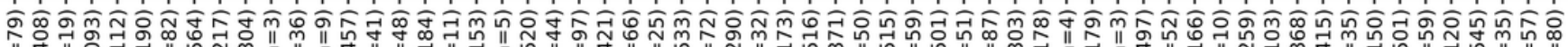
II

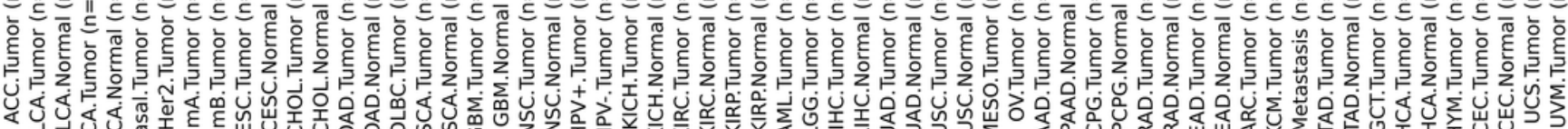

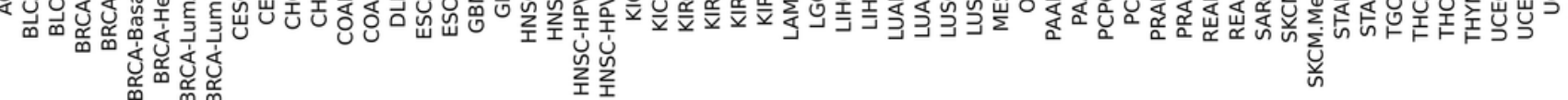

\section{Figure 1}

IKBIP expression in various types of cancers in TIMER database. ${ }^{\star} \mathrm{P}<0.05 ;{ }^{* \star} \mathrm{P}<0.01 ;{ }^{* \star * P}<0.001$.

A

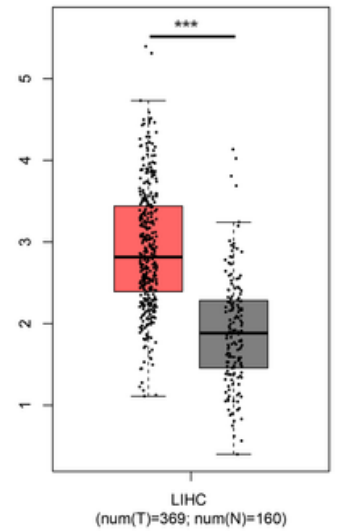

D

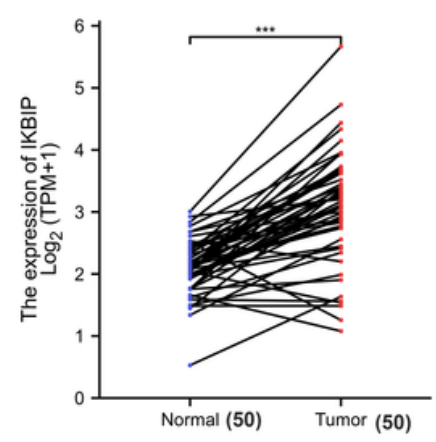

B

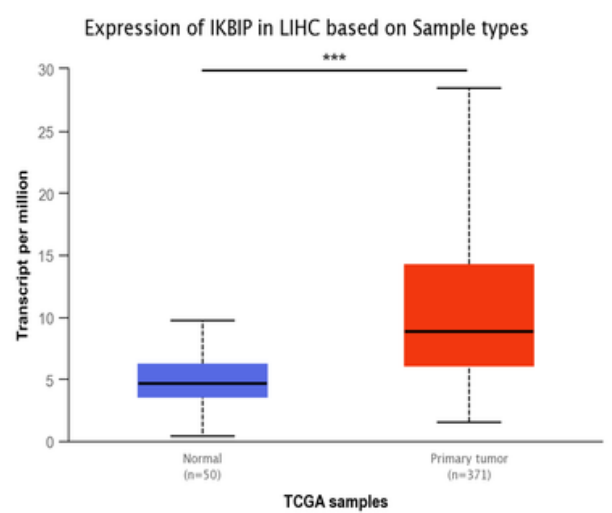

E

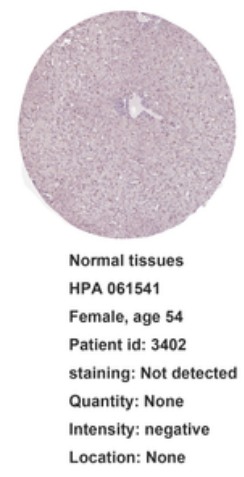

C

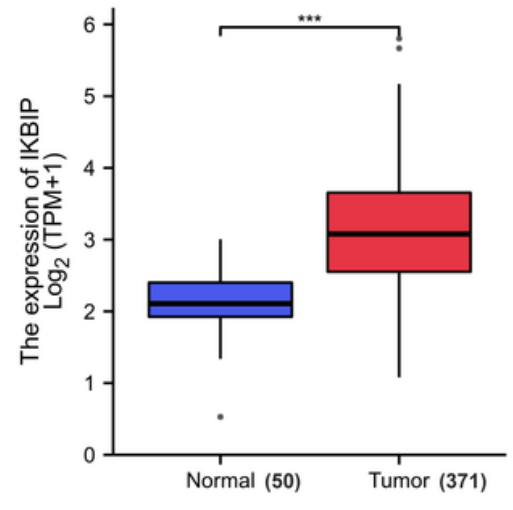

$\mathbf{F}$

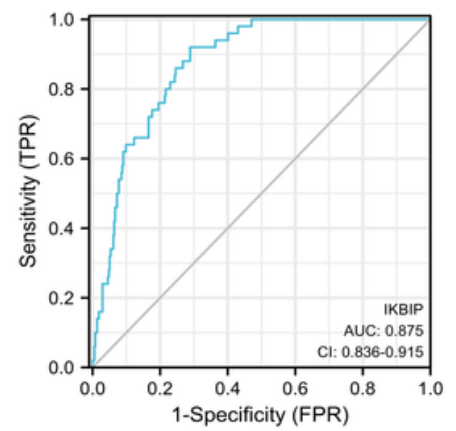

Figure 2 
IKBIP expression was elevated and correlated with diagnosis in HCC. (A) The level of IKBIP expression between normal and tumor tissues in GEPIA database, (B) The level of IKBIP expression between normal and tumor tissues in UALCAN database, (C) The level of IKBIP expression between normal and tumor tissues in the TCGA database, (D) The level of IKBIP expression in 50 HCC and matched-adjacent normal tissues in the TCGA database, (E) The protein level of IKBIP based on HPA, (F) The diagnostic value of IKBIP in HCC. ${ }^{* * *} \mathrm{P}<0.001$.

A

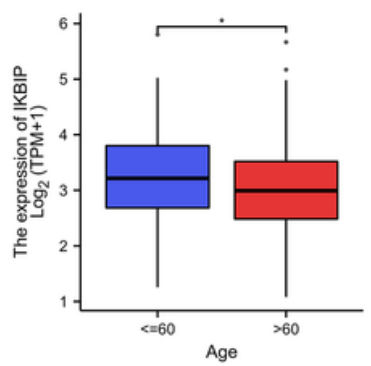

$E$

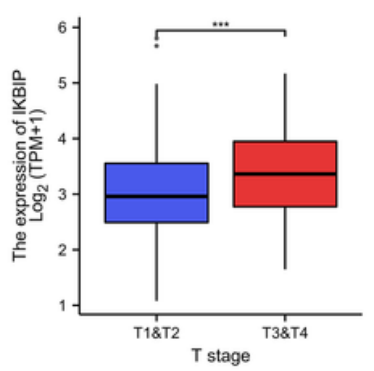

B

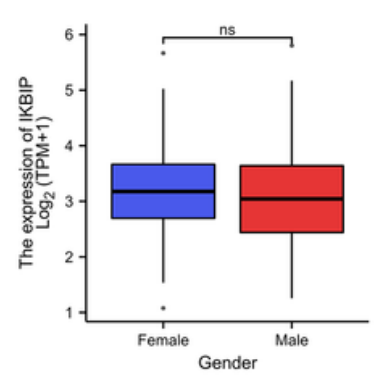

$\mathbf{F}$

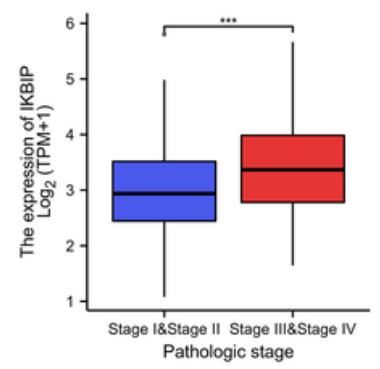

C

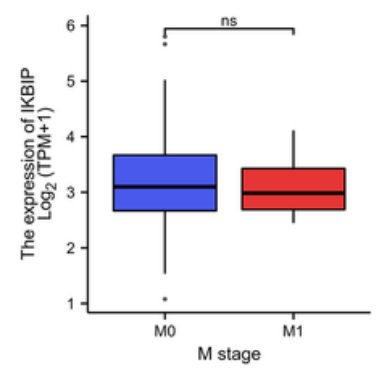

G

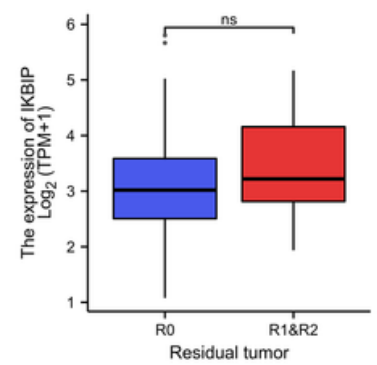

D

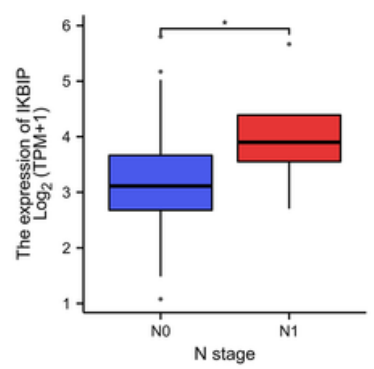

H

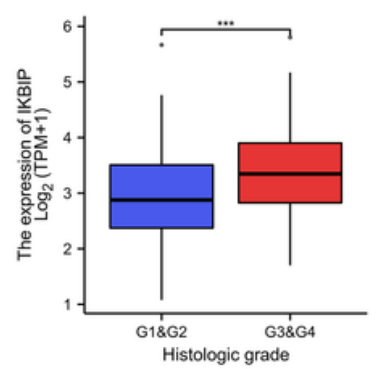

Figure 3

Correlations between IKBIP expression and clinical pathological features of HCC patients. (A) Age, (B) Gender, (C) M stage, (D) N stage, (E) T stage, (F) Pathologic stage, (G) Residual tumor, (H) Histologic grade. $n s P>0.05,{ }^{*} P<0.05, \star \star \star * P<0.001$. 
A

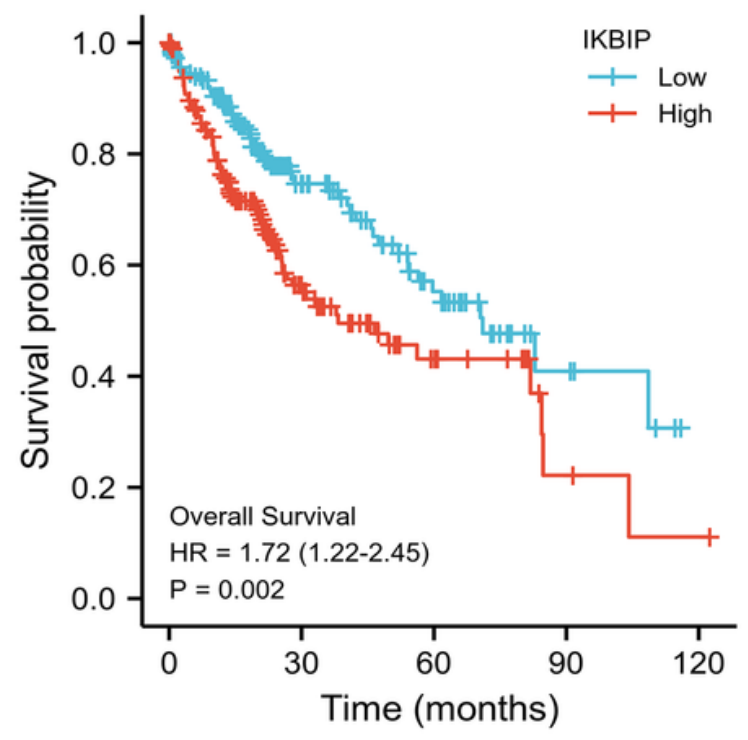

C Progression-Free Survival

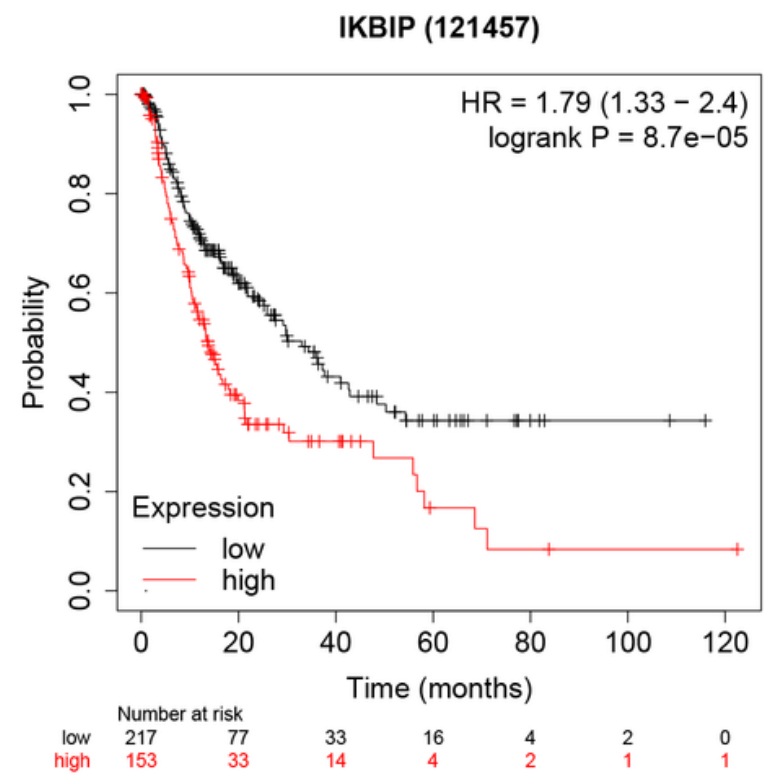

B

\section{Overall Survival}

IKBIP (121457)

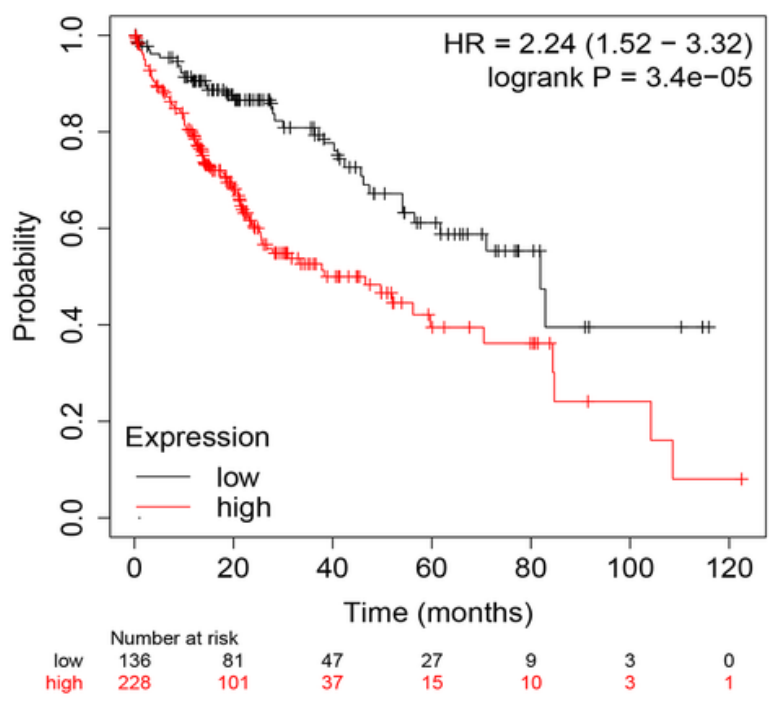

D Disease-Free Survival

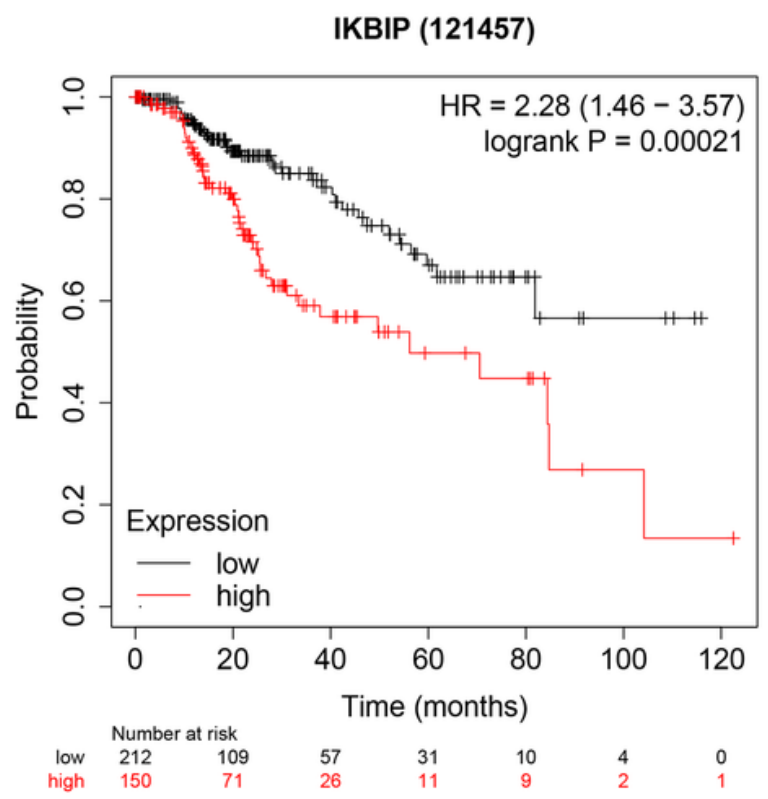

\section{Figure 4}

The prognostic potential of IKBIP expression in HCC patients. (A) The survival analysis of OS in TCGA database, (B) K-M plotter analysis of OS, (C) K-M plotter analysis of PFS, (D) K-M plotter analysis of DFS. 
A

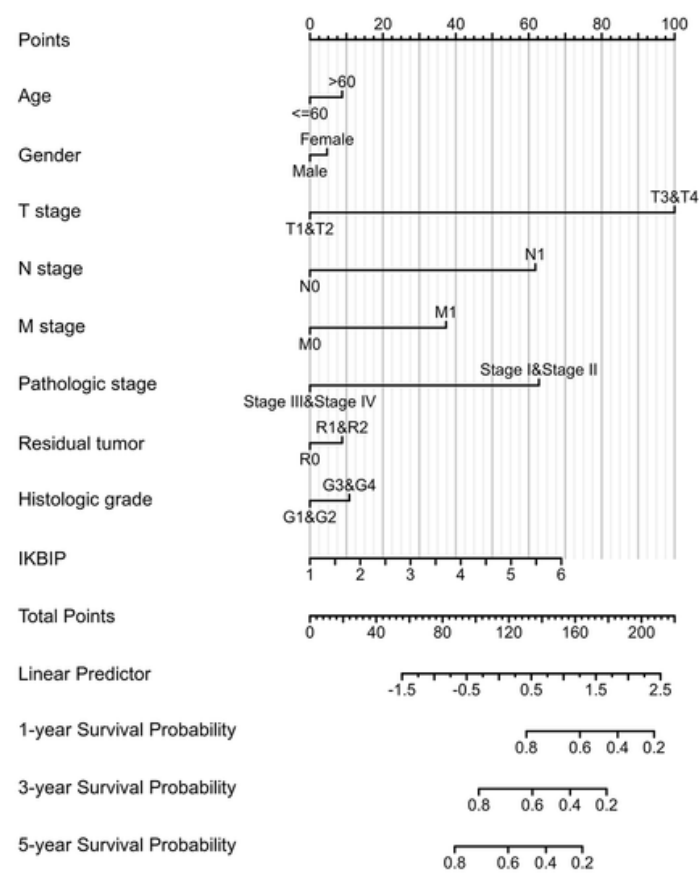

B

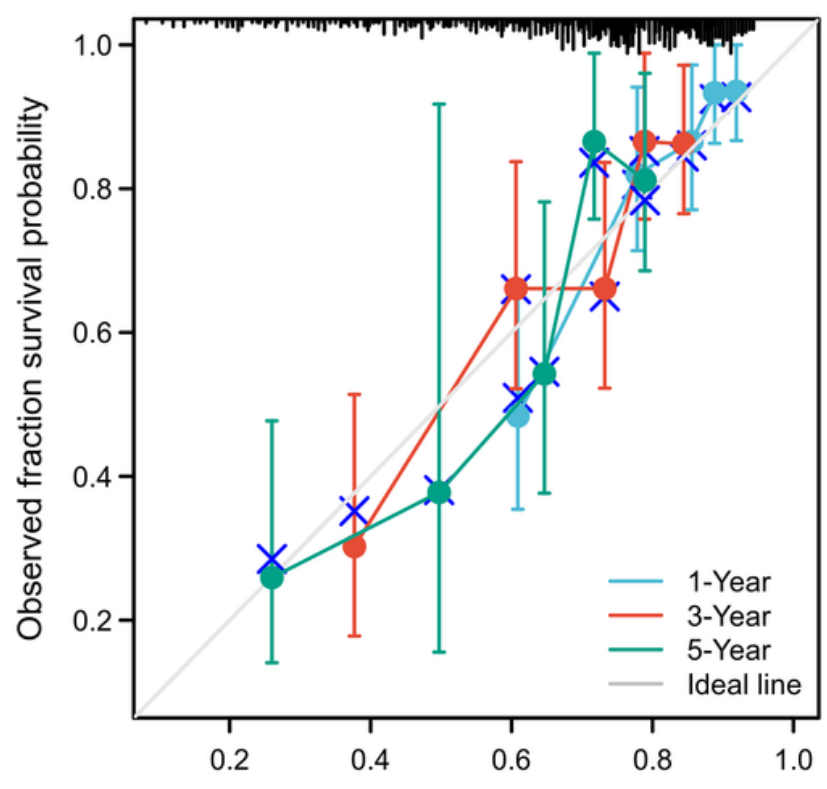

Nomogram prediced survival probability

\section{Figure 5}

The nomogram and calibration for predicting the 1-, 3- and 5-year OS of HCC patients. (A) Nomogram curve, (B) Calibration curve. 
A

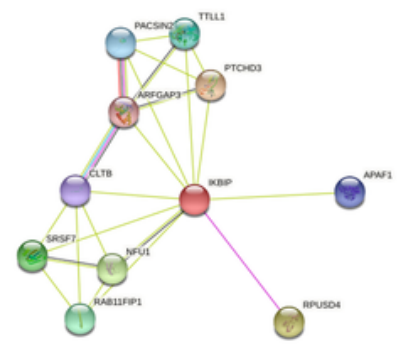

D

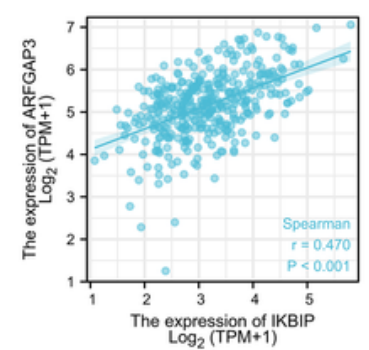

G

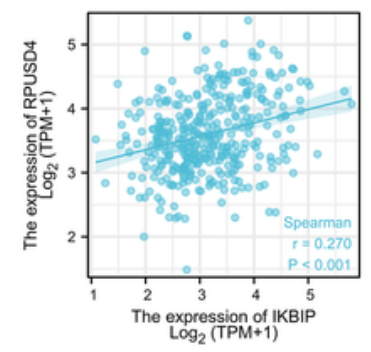

J

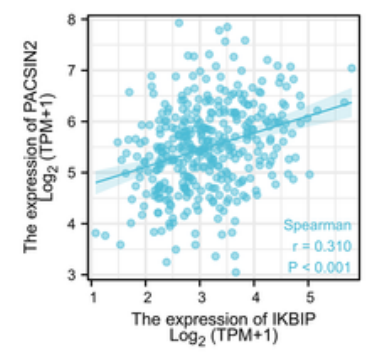

B

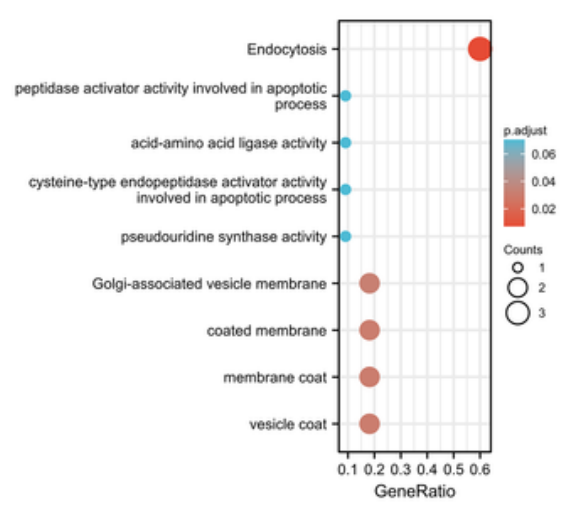

E

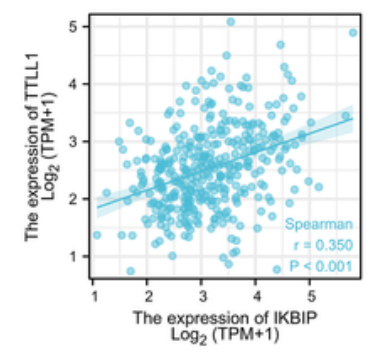

H

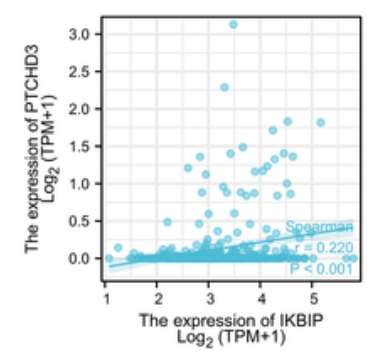

K

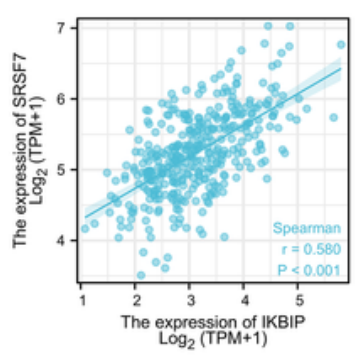

C

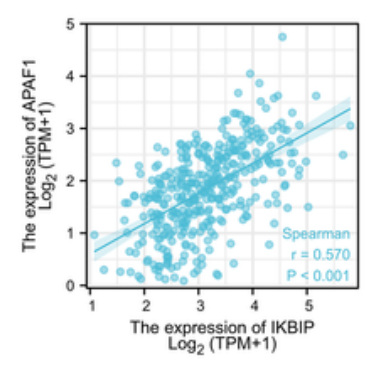

F

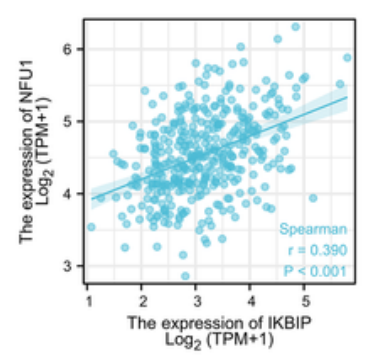

I

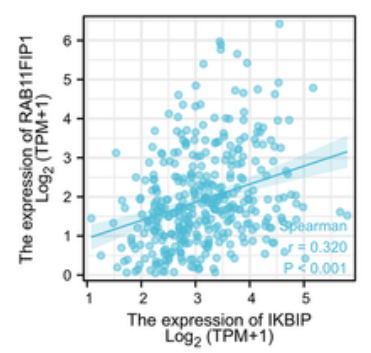

L

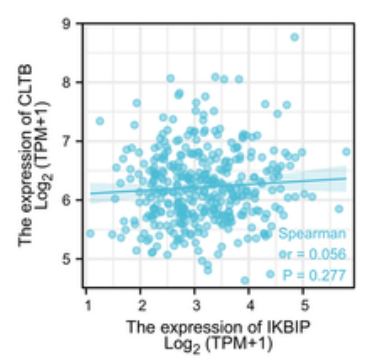

\section{Figure 6}

PPI networks and gene functional enrichment analysis. (A) The network of IKBIP and its co-expression genes, (B) GO and KEGG analyses of IKBIP and its co-expression genes, (C-K) The interrelation analyses between IKBIP expression and co-expressed genes in HCC. 
A
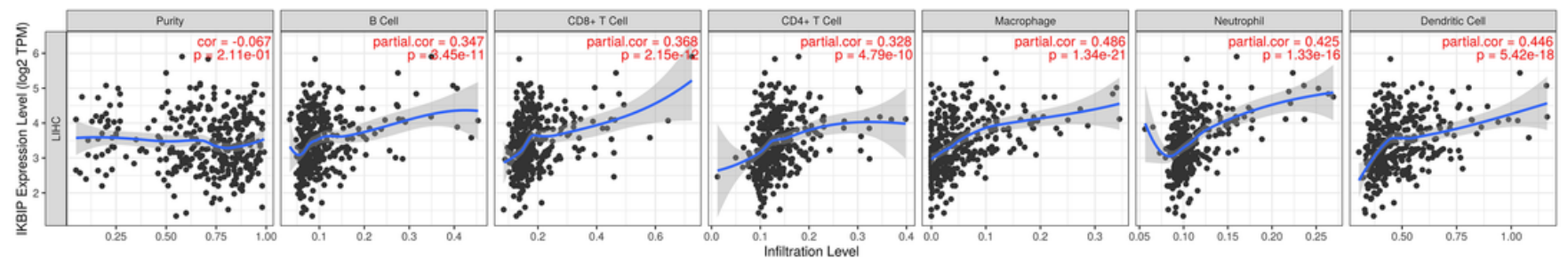

B

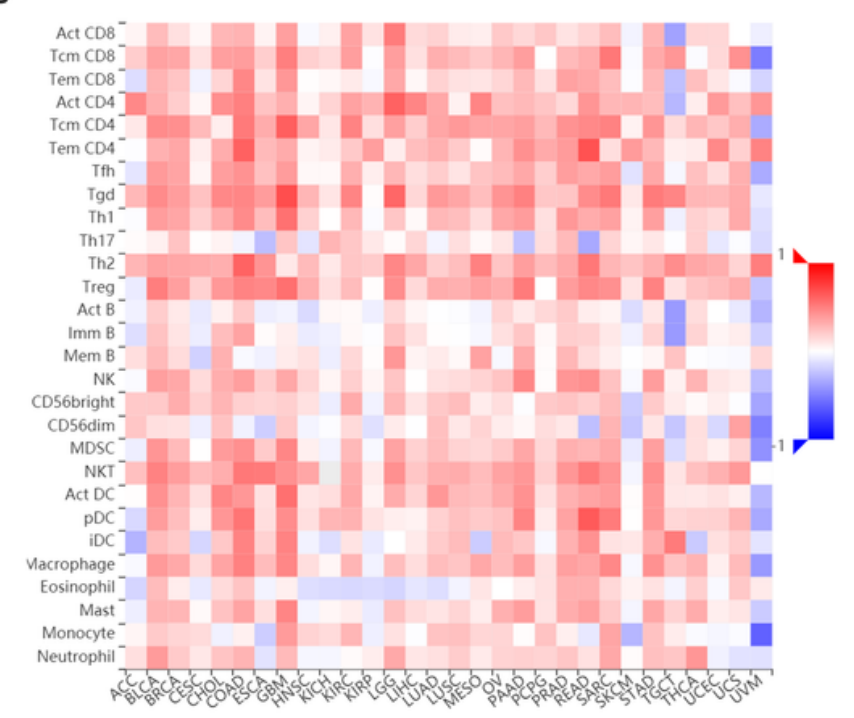

C
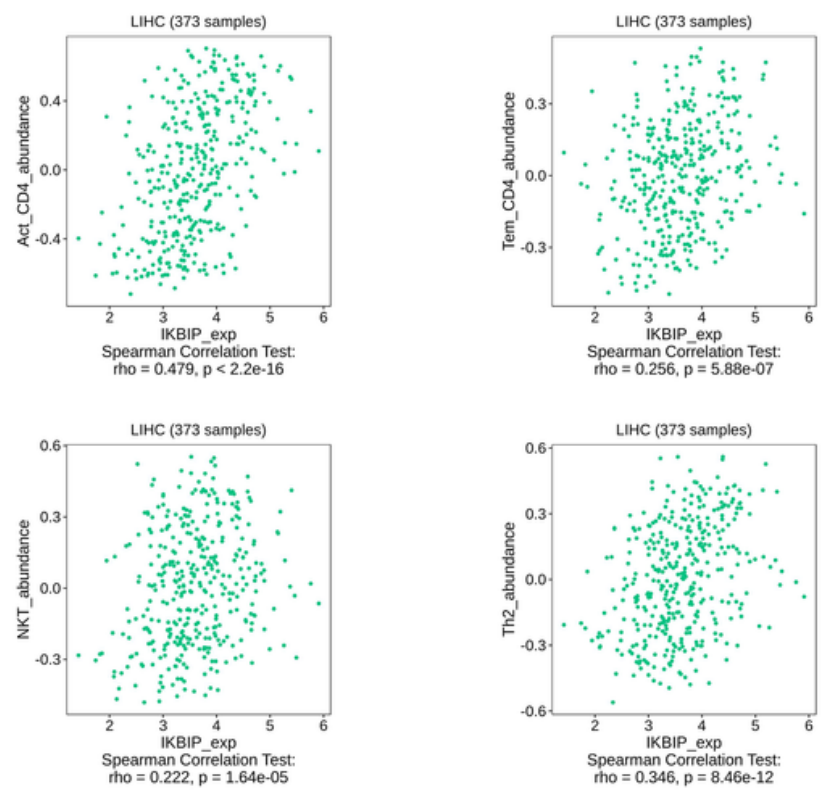

\section{Figure 7}

Correlation analysis between IKBIP expression and immune infiltrations. (A) Correlation between IKBIP expression and tumor purity, B cell, CD8+ T cell, CD4+ T cell, macrophage, neutrophil, dendritic cell in HCC, (B) Correlation between IKBIP expression and TILs in various cancers, (C) Correlation between IKBIP expression and Act-CD4+ T cells, Tem-CD4+ T cells, NKT cells and Th2 cells. 